\title{
Correction and Extension of van der Poel's Method for Calculating the Shear Modulus of a Particulate Composite
}

\author{
Jack C. Smith \\ Institute for Materials Research, National Bureau of Standards, Washington, D.C. 20234
}

(January 28, 1974)

\begin{abstract}
Van der Poel's method (Rheol. Acta 1, 198 (1958)) for calculating the shear modulus of a particulate composite agrees well with experimental data, but its validity has been questioned, and it was applicable only to composites in which the matrix material is incompressible. These limitations are removed in this paper in which an error in the original derivation is corrected, and the method generalized to apply to any matrix material. Calculations using the corrected theory show that despite the error, a table of shear modulus values published with the original theory is sufficiently correct for most practical purposes. Applicability of the generalized method to the large class of composites having compressible matrices is discussed. Shear moduli calculated by the corrected and extended method are compared with corresponding values calculated by other methods currently used.
\end{abstract}

Key words: Bulk modulus; composite materials; elastic constants; filled polymers; mechanical properties; particulate composites; shear modulus; theory of elasticity.

\section{Introduction}

Consider an idealized composite material consisting of small spheres imbedded in a matrix. The spheres are of approximately the same size, are firmly attached to the matrix, and are uniformly distributed so that the composite material is macroscopically homogeneous and isotropic. An important question then arises - that of calculating the two mechanical moduli characterizing this material in terms of the moduli characterizing the filler spheres and the matrix.

Several solutions have been given to this problem. The best known ones are those of Hashin and Shtrikman [1], ${ }^{1}$ Kerner [2], and Budiansky [3]. Hashin and Shtrikman's formulae, which provide upper and lower bounds for the shear and bulk moduli of the composite as a function of filler content, are: for the highest lower bound (HLB),

$$
\begin{gathered}
G=G_{m}+\frac{\varphi_{f}}{\frac{1}{G_{f}-G_{m}}+\frac{6\left(K_{m}+2 G_{m}\right) \varphi_{m}}{5 G_{m}\left(3 K_{m}+4 G_{m}\right)}} \\
K=K_{m}+\frac{\varphi_{f}}{\frac{1}{K_{f}-K_{m}}+\frac{3 \varphi_{m}}{3 K_{m}+4 G_{m}}}
\end{gathered}
$$

for the lowest upper bound (LUB),

\footnotetext{
${ }^{1}$ Figures in brackets indicate the literature references at the end of this paper.
}

$$
\begin{gathered}
G=G_{f}+\frac{\varphi_{m}}{\frac{1}{G_{m}-G_{f}}+\frac{6\left(K_{f}+2 G_{f}\right) \varphi_{f}}{5 G_{f}\left(3 K_{f}+4 G_{f}\right)}} \\
K=K_{f}+\frac{\varphi_{m}}{\frac{1}{K_{m}-K_{f}}+\frac{3 \varphi_{f}}{3 K_{f}+4 G_{f}}} .
\end{gathered}
$$

In these formulae $G$ represents the shear modulus, $K$ bulk modulus, and $\varphi$ volume fraction, the subscripts $f$ and $m$ refer to the filler and matrix respectively.

Kerner's formulae, although derived by a different method, can be shown by algebraic manipulation to be the same as those for Hashin and Shtrikman's highest lower bound $(1,2)$.

Budiansky provides the pair of coupled implicit equations:

$$
\begin{aligned}
& \frac{1}{G}=\frac{1}{G_{m}}+\left(1-\frac{G_{f}}{G_{m}}\right) \frac{\varphi_{f}}{G+\beta\left(G_{f}-G\right)} \\
& \frac{1}{K}=\frac{1}{K_{m}}+\left(1-\frac{K_{f}}{K_{m}}\right) \frac{\varphi_{f}}{K+\alpha\left(K_{f}-K\right)}
\end{aligned}
$$

where

$$
\alpha=\frac{3 K}{3 K+4 G}
$$

and

$$
\beta=\frac{6(K+2 G)}{5(3 K+4 G)}
$$


Young's modulus $E$ and Poisson's ratio $\nu$ can be calculated from the well known expressions:

$$
\begin{aligned}
& E=\frac{9 K G}{3 K+G} \\
& \nu=\frac{3 K-2 G}{6 K+2 G} .
\end{aligned}
$$

In van der Poel's solution [4], for which excellent agreement with experimental data is claimed [4,5], the formula for $K$ can be shown to be the same as eq (2), but the calculation for $G$ is too complicated to be expressed conveniently in closed form. Van der Poel has treated the special case in which the matrix of the composite has a Poisson's ratio $\nu=0.5$. The value of $G$ is obtained by interpolation from a table of values he has provided. It would be useful to have a formula applicable when the matrix has any value for Poisson's ratio. This was the purpose of the present work. During its progress an error was discovered in van der Poel's solution. The corrected form is given along with details of the derivation.

\section{Derivation}

The derivation is based on a method developed by Fröhlich and Sack [6].

To simplify calculations consider a unit length chosen such that on the average there is one sphere of filler in each spherical volume $4 \pi / 3$ of unit radius. If $a$ is the radius of the filler sphere, then by definition of the unit radius, $a^{3}$ is equal to the volume fraction $\varphi_{f}$ of the filler.

Consider a large cube of homogeneous material with edges parallel to the axes of a cartesian coordinate system having its origin at the center. Consider also a second cube in which the region at the center has the following structure: A filler sphere of radius $a$ is located at the origin. Surrounding this out to a radius unity is a sphere of matrix material which in turn is imbedded in the homogeneous material. The mechanical properties of this homogeneous material are assumed to be the same as the average macroscopic properties that are sought for the composite material considered here.

If the same boundary stresses are applied to each of the cubes, it is assumed that the displacements in the two cubes are the same at a distance $r=R \gg 1$ except for terms of a high order in $1 / r$. In the second cube the influence of the structure within the radius $r=1$ on the displacement at $r=R$ is expected to be of the same order as the ratio of the two volumes, i.e., $\sim 1 / R^{3}$. Thus it will be required that

Displacement at $r=R$ in second cube

Displacement at $r=R$ in first cube

$$
=1+\sum_{n>3} \frac{\text { const }}{R^{n}}
$$

Let the cube of homogeneous material (first cube) be subjected to the deviatoric stress system

$$
\sigma_{x}=\sigma_{y}=-T, \sigma_{z}=2 T, \tau_{x y}=\tau_{y z}=\tau_{z x}=0
$$

where the $\sigma s$ represent tensile and the $\tau s$ shear stresses. Assuming Hooke's law, it is easily seen that this stress system gives rise to the following displacements $u_{x}, u_{y}, u_{z}$ within the material;

$$
u_{x}=-\frac{T(1+\nu) x}{E}=-\frac{T x}{2 G}, u_{y}=-\frac{T y}{2 G}, u_{z}=\frac{T z}{G} .
$$

In spherical polar coordinates the components of stress and displacement are obtained by transformation as

$$
\begin{aligned}
& \sigma_{r}=2 T P_{2}(\operatorname{Cos} \theta), \tau_{r \theta}=T P_{2}^{\prime}(\operatorname{Cos} \theta), \tau_{r \varphi}=0 \\
& u_{r}=\frac{T r}{G} P_{2}(\operatorname{Cos} \theta), u_{\theta}=\frac{T r}{2 G} P_{2}^{\prime}(\operatorname{Cos} \theta), u_{\varphi}=0
\end{aligned}
$$

where $P_{2}(\operatorname{Cos} \theta)$ is the second Legendre polynomial and $P_{2}^{\prime}(\operatorname{Cos} \theta)$ is its derivative with respect to $\theta$.

$$
\begin{aligned}
& P_{2}(\operatorname{Cos} \theta)=\frac{1}{2}\left(3 \operatorname{Cos}^{2} \theta-1\right), \\
& P_{2}^{\prime}(\operatorname{Cos} \theta)=-3 \operatorname{Cos} \theta \operatorname{Sin} \theta .
\end{aligned}
$$

In order to find the displacements in the cube containing the special spherical structure at the center (second cube), it is necessary to find appropriate solutions to Lamé's equations expressed in spherical polar coordinates. These equations are tabulated by Love [7]. Because of symmetry of the stress system about the $z$ axis, the displacements $u_{r}$ and $u_{\theta}$ are independent of the azimuthal angle $\varphi$, and the displacement $u_{\varphi}=0$. Lamé's equations thus simplify to

$$
\begin{aligned}
& (\lambda+2 G) \operatorname{Sin} \theta \frac{\partial \Delta}{\partial \theta}+2 G \frac{\partial}{\partial r}(r \omega \varphi \operatorname{Sin} \theta)=0 \\
& (\lambda+2 G) r \operatorname{Sin} \theta \frac{\partial \Delta}{\partial r}-2 G \frac{\partial}{\partial \theta}(\omega \varphi \operatorname{Sin} \theta)=0
\end{aligned}
$$

where according to Love [8] the dilatation $\Delta$ is given by

$$
\Delta=\frac{1}{r^{2} \operatorname{Sin} \theta}\left\{\frac{\partial}{\partial r}\left(r^{2} u_{r} \operatorname{Sin} \theta\right)+\frac{\partial}{\partial \theta}\left(r u_{\theta} \operatorname{Sin} \theta\right)\right\}
$$

and the rotation $\omega_{\varphi}$ by

$$
\omega_{\varphi}=\frac{1}{2 r}\left\{\frac{\partial}{\partial r}\left(r u_{\theta}\right)-\frac{\partial}{\partial \theta} u_{r}\right\}
$$

Lamé's constant $\lambda$ is given by

$$
\lambda=\frac{2 G \nu}{1-2 \nu} .
$$


The most general solution having the same $P_{2}$ symmetry as the displacement system (15) is obtained by setting $u_{r}=r^{\beta} P_{2}(\operatorname{Cos} \theta), u_{\theta}=\alpha r^{\beta} P_{2}{ }^{\prime}(\operatorname{Cos} \theta)$ in eqs (17) to (19), and substituting for $\lambda$ using eq (20) to obtain the set of linear homogeneous equations in $\alpha$ :

$$
[(1-2 \nu)(\beta+1) \beta-12(1-\nu)] \alpha+[\beta+4(1-\nu)]=0
$$

$$
[-3 \beta+9-12 \nu] \alpha+[(1-\nu)(\beta+1) \beta-5+8 \nu]=0 .
$$

In order that these equations be compatible, it is necessary that

$$
\begin{array}{r}
\frac{\beta+4(1-\nu)}{(1-2 \nu)(\beta+1) \beta-12(1-\nu)} \\
=\frac{(1-\nu)(\beta+1) \beta-5+8 \nu}{-3 \beta+9-12 \nu}
\end{array}
$$

or, after simplification

$$
(\beta-1)(\beta+2)(\beta-3)(\beta+4)=0
$$

The value of $\alpha$ corresponding to each root of eq (22) is found by substituting into eq (21). The solutions for $u_{r}$ and $u_{\theta}$ thus become. ${ }^{2}$

$$
\begin{aligned}
& u_{r}=\left[A r+B r^{-4}+C r^{3}+D r^{-2}\right] P_{2}(\operatorname{Cos} \theta) \\
& u_{\theta}=\left[\frac{1}{2} A r-\frac{1}{3} B r^{-4}+b C r^{3}+c D r^{-2}\right] P_{2}^{\prime}(\operatorname{Cos} \theta)
\end{aligned}
$$

where

$$
b=\frac{7-4 \nu}{12 \nu}, c=\frac{1-2 \nu}{5-4 \nu}
$$

and $A, B, C, D$ are constants.

The strains $e_{r r}$ and $e_{r \theta}$ are found from the relations

$$
e_{r r}=\frac{\partial}{\partial r} u_{r}, e_{r \theta}=\frac{\partial}{\partial r} u_{\theta}-\frac{u_{\theta}}{r}+\frac{1}{r} \frac{\partial}{\partial \theta} u_{r}
$$

and the stresses necessary for the solution are then calculated using

$$
\sigma_{r}=\frac{2 G \nu}{1-2 \nu}+2 G e_{r r}, \tau_{r \theta}=G e_{r \theta}
$$

to obtain ${ }^{2}$

$$
\begin{aligned}
\sigma_{r} & =2 G\left[A-4 B r^{-5}+k_{1} C r^{2}+k_{3} D r^{-3}\right] P_{2}(\operatorname{Cos} \theta) \\
\tau_{r \theta} & =G\left[A+\frac{8}{3} B r^{-5}+k_{2} C r^{2}+k_{4} D r^{-3}\right] P_{2}^{\prime}(\operatorname{Cos} \theta)
\end{aligned}
$$

\footnotetext{
${ }^{2}$ For an incompressible material $(\nu=0.5)$ van der Poel's solution agrees with the solution obtained here, but when $\ddot{\nu} \neq 0.5$ the exponents in the terms having coefficients $C$ and $D$ in van der Poel's solution are erroneously given as functions of $\nu$. This error has previously been noted by Hashin [9].
}

where

$$
\begin{array}{r}
k_{1}=-\frac{1}{2}, k_{2}=\frac{7+2 \nu}{6 \nu}, k_{3}=-\frac{2(5-\nu)}{5-4 \nu}, \\
k_{4}=\frac{2(1+\nu)}{5-4 \nu} .
\end{array}
$$

Within the filler sphere $r \leqslant a$ the requirement that the displacement be zero at the origin necessitates that $B_{f}=D_{f}=0$, where the subscript $f$ refers to the filler medium. The solution applicable in the region $a \leqslant r \leqslant 1$ involves the constants $A_{m}, B_{m}, C_{m}, D_{m}$ where the subscript $m$ refers to the matrix medium. Within the region $1 \leqslant r \leqslant R$ requirement (11) necessitates that $A=T / G, C=D=0$. A must be regarded as an unknown constant as it involves the shear modulus $G$, the quantity which is to be calculated.

At the boundaries $r=a$ and $r=1$ the radial and tangential components of the displacement and the stress must be continuous. This gives rise to the following set of linear homogeneous equations in the unknowns $A_{f}, C_{f}, A_{m}, B_{m}, C_{m}, D_{m}, A, B$.

$$
\begin{array}{r}
M A_{f}-\frac{1}{2} M a^{2} C_{f}-A_{m}+4 a^{-5} B_{m}+\frac{1}{2} a^{2} C_{m}-k_{3 m} a^{-3} D_{m}=0 \\
M A_{f}+M k_{2 f} a^{2} C_{f}-A_{m}-\frac{8}{3} a^{-5} B_{m} \\
-k_{2 m} a^{2} C_{m}-k_{4 m} a^{-3} D_{m}=0 \\
a A_{f}+a^{3} C_{f}-a A_{m}-a^{-4} B_{m}-a^{3} C_{m}-a^{-2} D_{m}=0 \\
\frac{1}{2} a A_{f}+b_{f} a^{3} C_{f}-\frac{1}{2} a A_{m}+\frac{1}{3} a^{-4} B_{m} \\
-b_{m} a^{3} C_{m}-c_{m} a^{-2} D_{m}=0 .
\end{array}
$$

$$
\begin{aligned}
A_{m}-4 B_{m}-\frac{1}{2} C_{m}+k_{3 m} D_{m}+4 N B-N A & =0 \\
A_{m}+\frac{8}{3} B_{m}+k_{2 m} C_{m}+k_{4 m} D_{m}-\frac{8}{3} N B-N A & =0 \\
A_{m}+B_{m}+C_{m}+D_{m}-B-A & =0 \\
\frac{1}{2} A_{m}-\frac{1}{3} B_{m}+b_{m} C_{m}+c_{m} D_{m}+\frac{1}{3} B-\frac{1}{2} A & =0
\end{aligned}
$$

where

$$
M=G_{f} / G_{m}, N=G / G_{m}
$$

In order that the solution be nontrivial the determinant of the coefficients must be set equal to zero. Solving the resulting equation will evaluate the shear modulus of the material $G$, which is the quantity desired. 
After simplifications the determinantal equation becomes $^{3}$

$\begin{array}{|llllllll|}2 M & -2 M \nu_{f} & -2 & 8 & 2 \nu_{m} & 4\left(5-\nu_{m}\right) & 0 & 0 \\ 0 & 2 M\left(7+5 \nu_{f}\right) & 0 & -40 & -2\left(7+5 \nu_{m}\right) & -72 & 0 & 0 \\ 0 & -\left(7-10 \nu_{f}\right) & 0 & -5 & 7-10 \nu_{m} & -9 & 0 & 0 \\ 3 & 7-4 \nu_{J} & -3 & 2 & -\left(7-4 \nu_{m}\right) & -6\left(1-2 \nu_{m}\right) & 0 & 0 \\ 0 & 0 & 0 & 0 & -14\left(1-\nu_{m}\right) a^{-2} & 48\left(1-\nu_{m}\right) a^{3} & 8 X & 0 \\ 0 & 0 & 0 & 0 & 0 & -30\left(1-\nu_{m}\right) a^{3} & 0 & X \\ 0 & 0 & 1 & a^{5} & 2 \nu_{m} a^{-2} & \left(5-4 \nu_{m}\right) a^{3} & -1 & 0 \\ 0 & 0 & 5 & 0 & 7 a^{-2} & 4\left(4-5 \nu_{m}\right) a^{3} & 0 & 1\end{array}$

where $X=N-1=\frac{G}{G_{m}}-1$.

Equation (33) can be expanded into the form

$$
A X^{2}+B X+C=0
$$

where

$$
\begin{aligned}
A= & 2(M-1)\left[M\left(7+5 \nu_{f}\right)\right. \\
& \left.+4\left(7-10 \nu_{f}\right)\right]\left[4\left(7-10 \nu_{m}\right)\left(4-5 \nu_{m}\right)\left(1-a^{3}\right)\left(1-a^{7}\right)\right. \\
& \left.-63 a^{3}\left(1-a^{2}\right)^{2}\right] \\
& +280(M-1)\left(7-10 \nu_{f}\right)\left(1-\nu_{m}\right)\left(4-5 \nu_{m}\right)\left(1-a^{3}\right) a^{7} \\
& +60\left[M\left(7+5 \nu_{f}\right)\right. \\
& \left.+4\left(7-10 \nu_{f}\right)\right]\left(1-\nu_{m}\right)\left(7-10 \nu_{m}\right)\left(1-a^{7}\right) \\
& +2100\left(7-10 \nu_{f}\right)\left(1-\nu_{m}\right)^{2} a^{7} \\
B= & 70(M-1)\left[M\left(7+5 \nu_{f}\right)\right. \\
& \left.+4\left(7-10 \nu_{f}\right)\right]\left(1-\nu_{m}\right)\left[\left(4-5 \nu_{m}\right)\left(1-a^{3}\right)\right. \\
& \left.-6 a^{3}\left(1-a^{2}\right)\right] \\
& +525\left[M\left(7+5 \nu_{f}\right)+4\left(7-10 \nu_{f}\right)\right]\left(1-\nu_{m}\right)^{2} \\
& +60(M-1)\left[M\left(7+5 \nu_{f}\right)\right. \\
& \left.+4\left(7-10 \nu_{f}\right)\right]\left(1-\nu_{m}\right)\left[10 \nu_{m} a^{3}\left(1-a^{7}\right)\right. \\
& \left.-7 a^{5}\left(1-a^{5}\right)\right] \\
& -2100(M-1)\left(7-10 \nu_{f}\right)\left(1-\nu_{m}\right)^{2} a^{10} \\
C= & -525(M-1)\left[M\left(7+5 \nu_{f}\right)\right. \\
& \left.+4\left(7-10 \nu_{f}\right)\right]\left(1-\nu_{m}\right)^{2} a^{3} .
\end{aligned}
$$

One of the roots of eq (35) is negative and is extraneous. The positive root when substituted into eq (34) provides the value of the shear modulus $G$.

${ }^{3}$ The determinant given by van der Poel agrees with the determinant, eq (33), when
$\nu_{m}=0.5$ except for elements in the second column. These elements in van der Poel's solution are more complicated functions of $\nu_{f}$.

When the volume fraction $\varphi=a^{3}$ of the filler spheres is small, terms of higher order than $a^{3}$ can be neglected. The expressions for $A, B$, and $C$ become much shorter, and a further simplification results because the term $\left[M\left(7+5 \nu_{f}\right)+4\left(7-10 \nu_{f}\right)\right]$ can be factored from each of these quantities. The solution of eq (35) for this case yields the following approximate formula for $G$ :

$$
\frac{G}{G_{m}}=1+\frac{15\left(1-\nu_{m}\right)\left(G_{f}-G_{m}\right) \varphi}{\left(8-10 \nu_{m}\right) G_{f}+\left(7-5 \nu_{m}\right) G_{m}} .
$$

\section{Discussion}

It can be shown by algebraic manipulation that the formulas (39), (1), (5) and (3) for $G$ can be displayed in the form

$$
G=G_{m}+\left(G_{f}-G_{m}\right) \Phi=(1-\Phi) G_{m}+\Phi G_{f}
$$

which, when $\Phi=\varphi$, is the well-known rule of mixtures. For the approximate van der Poel equation (39)

$$
\Phi=\frac{\left(8-10 \nu_{m}\right) G_{m}+\left(7-5 \nu_{m}\right) G_{m}}{\left(8-10 \nu_{m}\right) G_{f}+\left(7-5 \nu_{m}\right) G_{m}} \varphi
$$

For Kerner, or Hashin and Shtrikman HLB, (1)

$$
\Phi=\frac{\left(8-10 \nu_{m}\right) G+\left(7-5 \nu_{m}\right) G_{m}}{\left(8-10 \nu_{m}\right) G_{f}+\left(7-5 \nu_{m}\right) G_{m}} \varphi .
$$

For Budiansky (5)

$$
\Phi=\frac{(8-10 \nu) G+(7-5 \nu) G}{(8-10 \nu) G_{f}+(7-5 \nu) G} \varphi .
$$

For Hashin and Shtrikman LUB (3)

$$
\Phi=\frac{\left(8-10 \nu_{f}\right) G+\left(7-5 \nu_{f}\right) G_{f}}{\left(8-10 \nu_{f}\right) G_{f}+\left(7-5 \nu_{f}\right) G_{f}} \varphi
$$


This display shows that these formulas are all of the same type, and can be interchanged into each other in a certain predictable way.

When the volume fraction of filler $\varphi$ is very small so that $G \sim G_{m}$, and $\nu \sim \nu_{m}$, the values of $\Phi / \varphi$ computed by eqs (41) to (43) are essentially equal. Thus plots of $\Phi=\left(G-G_{m}\right) /\left(G_{f}-G_{m}\right)$ versus $\varphi$ for these relations all have the same initial slope. Plots of $G / G_{m}$ for these relations also have the same initial slope. Thus the initial increases of $G / G_{m}$ with increasing $\varphi$, as calculated by the methods of Hashin and Shtrikman HLB, Kerner, van der Poel, and Budiansky, are the same.

The slope $G / G_{m}$ computed from the approximate van der Poel formula (39) remains constant as $\varphi$ increases, but the slope computed by the accurate method (35) is nonlinear because of the complicated dependence on various powers of $a$ the filler sphere radius. The slopes computed using the formula of Kerner, or Hashin and Shtrikman HLB, (1) and Budiansky $(5,6)$ are also nonlinear, and increase with increasing $\varphi$.

When $\nu_{m}=0.5$ and $G_{f} / G_{m} \gg 1$ as in the case of a gum rubber matrix loaded with rigid filler particles, the shear modulus for small values of $\varphi$ should obey the equation

$$
G=G_{m}(1+2.5 \varphi) .
$$

This equation is analogous to an equation developed by Einstein [10] for the viscosity of a suspension of rigid spheres in a fluid medium, and has been used to predict reinforcement effects in rubber [11]. Under similar conditions the van der Poel formulae developed here (35), (39) and the formulae of Kerner, or Hashin and Shtrikman HLB, (1) and Budiansky $(5,6)$ reduce to eq (45). In fact the approximate formula (39) may be considered as a generalized form of eq (45) in which $G_{f}$ and $\nu_{m}$ are specified in addition to $G_{m}$.

In order to calculate $G$ using the formula of Kerner, or Hashin and Shtrikman HLB, (1), it is necessary to know only one of the moduli characterizing the filler material, i.e., $G_{f}$. This is in contrast to Budiansky's formulae $(5,6)$, where elimination of $K$ leads to a relationship for $G$ involving filler moduli $G_{f}$ and $K_{f}$. Similarly for the corrected van der Poel computation (35), two filler moduli $G_{f}$ and $\nu_{f}$ are required. The corrected van der Poel computation (35) and the formulae of Budiansky $(5,6)$ thus provide some indication of the relative effectiveness of the distortional (shear modulus) and dilatational (bulk modulus) properties of the filler in determining the shear modulus of the composite. To determine this relative effectiveness, computations were made by the two methods and the results are presented in tables 1 and 2.

In table 1 values of $G / G_{m}$ calculated from Budiansky's formulae $(5,6)$ are given, corresponding to various values of filler Poisson's ratio $\nu_{f}$ and the ratio $G_{f} / G_{m}$, for a matrix Poisson's ratio $\nu_{m}=0.5$. In the calculations the ratio of the shear or rigidity modulus of the filler to that of the matrix $G_{f} / G_{m}$ ranges in value from 10 to
TABLE 1. G/G $\mathrm{G}_{\mathrm{m}}$ for various values of $\nu_{\mathrm{f}}$ and $\mathrm{G}_{\mathrm{f}} / \mathrm{G}_{\mathrm{m}}$, calculated according to Budiansky's formulae $(5,6)$

\begin{tabular}{c|c|c|c|c|c}
\hline \hline & $\begin{array}{r}G / G_{m} \\
\text { corre- } \\
\text { sponding } \\
\text { to } \\
\nu_{f}\end{array}$ & 100 & 1,000 & 10,000 & 100,000 \\
& & & & \\
\hline \multicolumn{6}{c}{$\varphi=0.25, \nu_{m}=0.50$} \\
\hline 0.10 & 1.7759 & 2.4647 & 2.6428 & 2.6642 & 2.6664 \\
.30 & 1.7861 & 2.4726 & 2.6440 & 2.6644 & 2.6664 \\
.50 & 1.7958 & 2.4787 & 2.6449 & 2.6644 & 2.6664 \\
\hline \multicolumn{5}{c}{$\varphi=0.50, \nu_{m}=0.50$} \\
\hline 0.10 & 3.4855 & 17.871 & 146.56 & 1426.2 & 14221. \\
.30 & 3.5647 & 19.003 & 158.64 & 1548.6 & 15448. \\
.50 & 3.6565 & 20.143 & 170.74 & 1670.8 & 16671. \\
\hline
\end{tabular}

TABLE 2. G/ $\mathrm{G}_{\mathrm{m}}$ for various values of $\nu_{\mathrm{f}}$ and $\mathrm{G}_{\mathrm{f}} / \mathrm{G}_{\mathrm{m}}$, calculated according to the corrected van der Poel equation (35)

\begin{tabular}{c|c|c|c|c|c}
\hline \hline & $\begin{array}{r}G / G_{m} \\
\text { corre- } \\
\text { sponding } \\
\text { to } \\
G_{f} / G_{m}=10\end{array}$ & 100 & 1,000 & 10,000 & 100,000 \\
& & & & \\
\hline \multicolumn{6}{c}{$\varphi=0.25, \nu_{m}=0.50$} \\
\hline .10 & 1.6842 & 2.0025 & 2.0490 & 2.0540 & 2.0545 \\
.30 & 1.6843 & 2.0026 & 2.0491 & 2.0540 & 2.0545 \\
.50 & 1.6845 & 2.0027 & 2.0491 & 2.0540 & 2.0545 \\
\hline \multicolumn{5}{c}{$\varphi=0.50, \nu_{m}=0.50$} \\
\hline .10 & 3.1083 & 6.4334 & 7.8687 & 8.0738 & 8.0952 \\
.30 & 3.1235 & 6.4888 & 7.8810 & 8.0751 & 8.0953 \\
.50 & 3.1393 & 6.5356 & 7.8909 & 8.0762 & 8.0954 \\
\hline
\end{tabular}

$10^{5}$, and from eq (10), the range of $\nu_{f}$ from 0.1 to 0.5 corresponds to a range of the bulk modulus $K_{f}$ from $(11 / 12) G_{f}$ to $\infty$. The tabulated values show that the relative shear modulus $G / G_{m}$ (Budiansky) can be strongly dependent upon the rigidity of the filler relative to the matrix $G_{f} / G_{m}$, but is only slightly sensitive to the value of the filler Poisson's ratio $\nu_{f}$ or the bulk modulus $K_{f}$.

Table 2 presents similar results calculated using the corrected van der Poel formula (35). Here again the values of the relative modulus $G / G_{m}$ are seen to be insensitive to the value of the filler Poisson's ratio. Thus although the Budiansky and corrected van der Poel formulas make use of the value of filler Poisson's ratio in the calculations of $G / G_{m}$, the values obtained are insensitive to it, and in this respect these formulas offer little advantage over the formula of Kerner, or Hashin and Shtrikman HLB, in which the value of filler Poisson's ratio is not needed.

Table 3 presents values of $G / G_{m}$ for various filler volume fractions $\varphi$ and values of $G_{f} / G_{m}$, computed according to the corrected van der Poel equation (35). In these calculations Poisson's ratio of the filler spheres was $\nu_{f}=0.25$, and of the matrix was $\nu_{m}=0.5$. If these values are divided by $G_{f} / G_{m}$ the resulting values are almost identical with the values of $G / G_{f}$ given in a 
TABLE 3. G/ $G_{m}$ as a function of $\varphi$ for various values of $G_{f} / G_{m}$, calculated according the corrected van der Poel equation (35)

\begin{tabular}{|c|c|c|c|c|c|c|c|}
\hline \multicolumn{8}{|c|}{$\nu_{m}=0.50, \nu_{f}=0.25$} \\
\hline$\varphi$ & $\begin{array}{c}G / G_{m} \\
\text { corresponding to } \\
G_{f} / G_{m}=2\end{array}$ & 10 & 20 & 100 & 1,000 & 10,000 & 100,000 \\
\hline .00 & 1.0000 & 1.0000 & 1.0000 & 1.0000 & 1.0000 & 1.0000 & 1.0000 \\
\hline .05 & 1.0363 & 1.1025 & 1.1166 & 1.1296 & 1.1328 & 1.1331 & 1.1332 \\
\hline .10 & 1.0738 & 1.2177 & 1.2504 & 1.2814 & 1.2891 & 1.2899 & 1.2900 \\
\hline .15 & 1.1126 & 1.3499 & 1.4087 & 1.4665 & 1.4813 & 1.4828 & 1.4829 \\
\hline .20 & 1.1529 & 1.5038 & 1.6000 & 1.6998 & 1.7262 & 1.7289 & 1.7292 \\
\hline .25 & 1.1946 & 1.6843 & 1.8350 & 2.0026 & 2.0491 & 2.0540 & 2.0545 \\
\hline .30 & 1.2379 & 1.8962 & 2.1264 & 2.4066 & 2.4901 & 2.4991 & 2.5000 \\
\hline .35 & 1.2825 & 2.1438 & 2.4888 & 2.9606 & 3.1162 & 3.1333 & 3.1350 \\
\hline .40 & 1.3286 & 2.4301 & 2.9376 & 3.7398 & 4.0447 & 4.0798 & 4.0834 \\
\hline .45 & 1.3761 & 2.7559 & 3.4852 & 4.8580 & 5.4924 & 5.5713 & 5.5794 \\
\hline .50 & 1.4249 & 3.1196 & 4.1376 & 6.4759 & 7.8782 & 8.0748 & 8.0953 \\
\hline .55 & 1.4750 & 3.5174 & 4.8898 & 8.7861 & 12.031 & 12.578 & 12.636 \\
\hline .60 & 1.5264 & 3.9448 & 5.7280 & 11.948 & 19.569 & 21.236 & 21.423 \\
\hline .65 & 1.5791 & 4.3986 & 6.6336 & 15.978 & 33.437 & 38.824 & 39.482 \\
\hline .70 & 1.6332 & 4.8794 & 7.5946 & 20.684 & 58.323 & 76.204 & 78.710 \\
\hline .75 & 1.6889 & 5.3937 & 8.6140 & 25.740 & 99.923 & 159.63 & 170.20 \\
\hline .80 & 1.7463 & 5.9570 & 9.7226 & 30.888 & 160.66 & 356.81 & 408.82 \\
\hline
\end{tabular}

similar table by van der Poel [4]. The elements in the second column of the determinantal equation evaluated by van der Poel involved different functions of $\nu_{f}$ than those given in eq (33) of this paper. Evidently van der Poel's results agree with those given in table 3 because of the insensitivity of the method of calculation to values of $\nu_{f}$.

The way in which results of the corrected van der Poel calculation compare with results calculated by the other methods considered here is illustrated in figures 1 and 2 . In the example chosen for figure 1 , Poisson's ratio of the matrix is $\nu_{m}=0.4$ and the ratio of filler to matrix rigidity is $G_{f} / G_{m}=30$. For those cases in which it is used, the value of Poisson's ratio of the filler is $\nu_{f}=0.25$. The plots of relative shear modulus $G / G_{m}$ versus $\varphi$ represent the expected shear properties of a series of particulate composites consisting of small glass spheres imbedded in a rigid epoxy matrix.

In the example depicted in figure $2, \nu_{m}=0.5$ and $G_{f} / G_{m}=70,000$. When used, $\nu_{f}=0.25$. The plots in this case represent the expected shear properties of a series of particulate composites consisting of small glass spheres imbedded in a lightly vulcanized matrix of natural rubber.

For the epoxy matrix composite, figure 1, the predictions of the van der Poel and Kerner, or Hashin and Shtrikman HLB, equations are about the same, but for the rubber matrix composite, figure 2 , the predictions of these two formulas are much different. The Budiansky calculation predicts the highest values of $G / G_{m}$ for both examples.

From assumptions made in the derivation, one should only expect reliable predictions from the van der Poel calculation when the volume fraction of the filler $\varphi$ is small. However, agreement of the theory with experimental results appears to be good even for large values of the volume fraction of filler. According to van der Poel [4], calculations for the case

$$
G_{f} / G_{m}=100,000, \nu_{f}=0.25, \nu_{m}=0.5
$$

are in good agreement for values of $\varphi$ up to 0.60 with Eilers' [12] experimental values for the relative viscosity of suspensions of bitumin particles in water.

If the values in table 3 corresponding to these van der Poel calculations are compared with Eilers' data, the same degree of agreement is found. In table 3 there is only slight difference between the values for $G_{f} / G_{m}=10^{4}$ and $G_{f} / G_{m}=10^{5}$. Therefore the curve in figure 2 of $G / G_{m}$ versus $\varphi$, calculated using $G_{f} / G_{m}$ $=70,000$ in the corrected van der Poel formula (35), lies very close to a similar curve that could be plotted from Eilers' data. The curves plotted using Budiansky's formulae and the Kerner, or Hashin and Shtrikman HLB, equation are quite different. It seems then that either eq (35) or van der Poel's original calculation provides the best agreement with Eilers' data.

Schwarzl [5] has studied the mechanical characteristics of composites consisting of $\mathrm{NaCl}$ crystals in a rubbery polyurethane matrix. He reports agreement for volume fractions of filler up to $\varphi=0.50$ between his results and van der Poel's theory using the parameter values $G_{f} / G_{m}=10^{4}, \nu_{f}=0.25$, and $\nu_{m}=0.50$. The curve in figure 2 calculated from the corrected van der Poel equation provides a good fit to Schwarzl's data, and the other two curves do not fit.

When measurements were made at low temperature so that the polyurethane matrix was in the glassy state, Schwarzl obtained data that could be fit by van der Poel's theory using the parameter values $G_{f} / G_{m}=8.4, \nu_{f}=0.25$, and $\nu_{m}=0.5$. The value of $\nu_{m}=0.5$ was used because van der Poel's table had been calculated for this value only. The value of $\nu_{m}$ that should have been used was probably close to $\nu_{m}=0.4$. This suggests that values of $G / G_{m}$ calculated by the van der Poel method are not sensitive to the value of the matrix Poisson's ratio $\nu_{m}$, so calculations were made to check this.

In table 4 values of $G / G_{m}$ calculated from the corrected van der Poel equation (35) are given, corresponding to various values of matrix Poisson's ratio $\nu^{\prime} m$ 


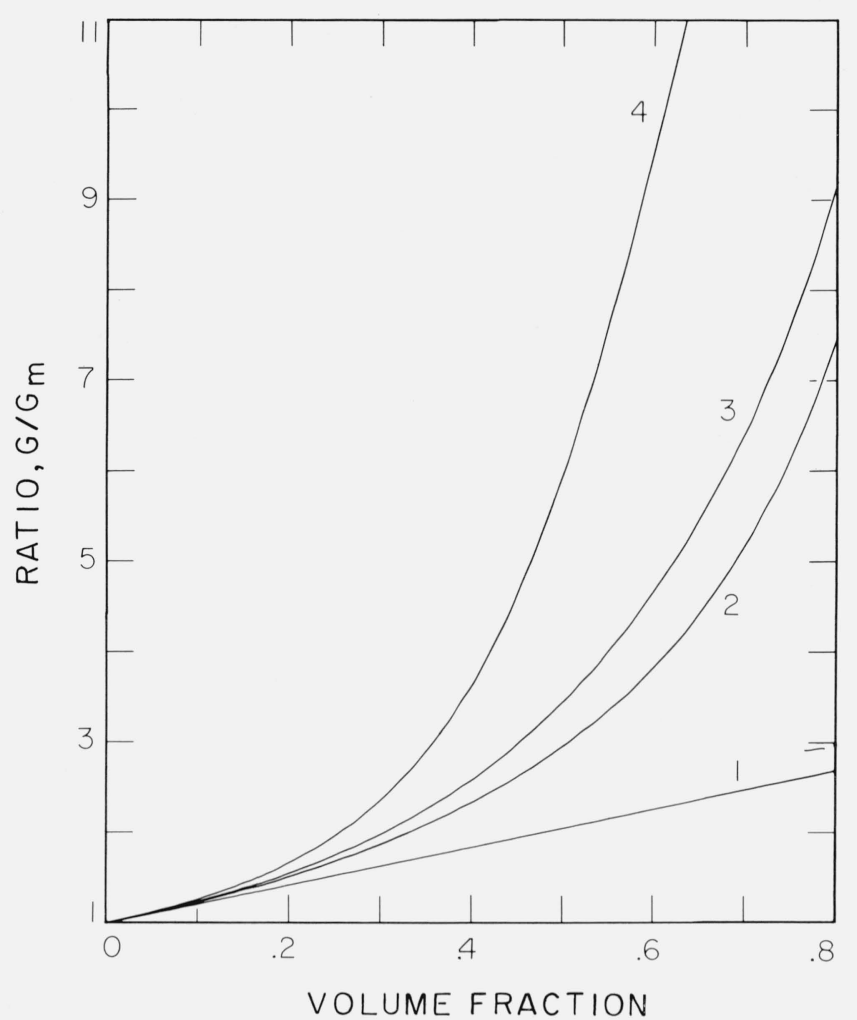

Figure 1. Plot of relative shear modulus $\mathrm{G} / \mathrm{G}_{\mathrm{m}}$ versus volume fraction of filler for the case $\mathrm{G}_{\mathrm{f}} / \mathrm{G}_{\mathrm{m}}=30, \nu_{\mathrm{f}}=0.25, \nu_{\mathrm{m}}=0.40$.

Curve 1, Approximate van der Poel equation (39); 2, equation of Kerner, or Hashin and Shtrikman HLB, (1); 3, corrected van der Poel equation (35); 4, Budiansky's formulae $(5,6)$.

TABLE 4. $\mathrm{G} / \mathrm{G}_{\mathrm{m}}$ for various values of $\nu_{\mathrm{m}}$ and $\mathrm{G}_{\mathrm{f}} / \mathrm{G}_{\mathrm{m}}$, calculated according to the corrected van der Poel equation (35)

\begin{tabular}{c|c|c|c|c|c|c}
\hline \hline$\nu_{m}$ & $\begin{array}{c}G / G_{m} \\
\text { corre- } \\
\text { sponding to } \\
G_{f} / G_{m}=3\end{array}$ & 10 & 30 & 100 & 1,000 & 10,000 \\
\hline \multicolumn{7}{c}{$\varphi=0.25, \nu_{f}=0.25$} \\
\hline 0.30 & 1.2955 & 1.5556 & 1.6747 & 1.7245 & 1.7451 & 1.7472 \\
.40 & 1.3058 & 1.5992 & 1.7445 & 1.8077 & 1.8343 & 1.8371 \\
.50 & 1.3224 & 1.6843 & 1.8982 & 2.0026 & 2.0491 & 2.0540 \\
\hline \multicolumn{7}{c}{$\varphi=0.50, \nu_{f}=0.25$} \\
\hline 0.30 & 1.6928 & 2.5562 & 3.0870 & 3.3470 & 3.4626 & 3.4747 \\
.40 & 1.7156 & 2.7189 & 3.4400 & 3.8359 & 4.0231 & 4.0432 \\
.50 & 1.7542 & 3.1197 & 4.7709 & 6.4759 & 7.8782 & 8.0748 \\
\hline
\end{tabular}

and the ratio $G_{f} / G_{m}$, for a filler Poisson's ratio $\nu_{f}=0.25$. These calculations show that when $G_{f} / G_{m} \sim 10$, changing $\nu_{m}$ from 0.5 to 0.4 changes the value of $G / G_{m}$ obtained only by a small amount, which approximates the experimental error expected in a measurement. When $G_{f} / G_{m} \geqslant 30$, the change in the calculated value of $G / G_{m}$ is significant, so that values of $G / G_{m}$ taken from a table calculated assuming $\nu_{m}=0.5$ would not provide a good fit to experimental data. Thus

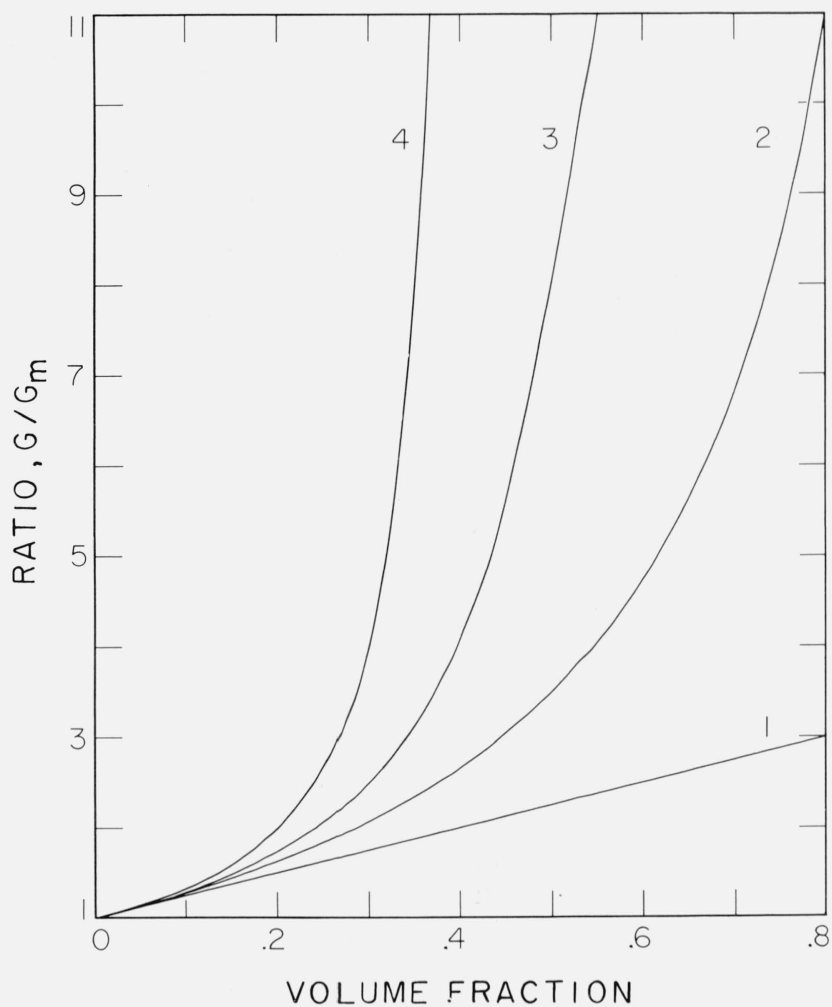

FigURE 2. Plot of relative shear modulus $\mathrm{G} / \mathrm{G}_{\mathrm{m}}$ versus volume fraction of filler for the case $\mathrm{G}_{\mathrm{f}} / \mathrm{G}_{\mathrm{m}}=70,000, \nu_{\mathrm{f}}=0.25, \nu_{\mathrm{m}}=0.50$.

Curve 1, Approximate van der Poel equation (39); 2, equation of Kerner, or Hashin and Shtrikman HLB, (1); 3, corrected van der Poel equation (35); 4, Budiansky's formulae $(5,6)$.

although Schwarzl was able to fit his low temperature data using van der Poel's table, the corrected and extended van der Poel equation (35) would be needed to fit data for a system in which $G_{f} / G_{m} \sim 30, \nu_{m}=0.4$ such as glass spheres imbedded in a rigid epoxy matrix.

\section{References}

[1] Hashin, Z., and Shtrikman, S., J. Mech. Phys. Solids 11, 127 (1963).

[2] Kerner, E. H., Proc. Phys. Soc. B69, 808 (1956).

[3] Budiansky, B., J. Mech. Phys. Solids 13, 223 (1965).

[4] van der Poel, C., Rheol. Acta 1, 198 (1958).

[5] Schwarzl, F. R., Mechanics and Chemistry of Solid Propellants, Proceedings of the Fourth Symposium on Naval Structural Mechanics, edited by A. C. Eringen, H. Liebowitz, S. L. Koh, and J. M. Crowley, (Pergamon Press, New York, N.Y., 1967), p. 503.

[6] Fröhlich, H. and Sack, R., Proc. Roy. Soc. A185, 415 (1946).

[7] Love, A. E. H., A Treatise on the Mathematical Theory of Elasticity, 4th ed. (Dover, 1941), p. 141.

[8] Love, A. E. H., loc cit, p. 56.

[9] Hashin, Z., Int. J. Solids Structures 6, 539 (1970).

[10] Einstein, A., Ann. Physik 17, 549 (1905); 19, 289 (1906); 34, 591 (1911).

[11] Nielsen, L. E., J. Comp. Matls. 1, 100 (1967).

[12] Eilers, H., Kolloid Z. 97, 313 (1941).

(Paper 78A3-816) 